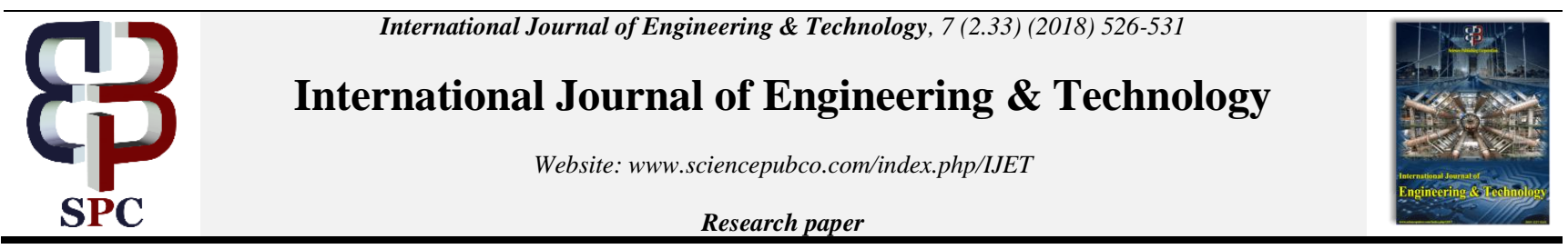

\title{
Optimization of fuzzy image pattern matching using genetic algorithm
}

\author{
Ravikumar D ${ }^{1 *}$, Dr. Arun Raaza ${ }^{2}$, Dr. V. Devi ${ }^{3}$ \\ ${ }^{1}$ Research Scholar, Department of Electronics and communication Engineering, Vels Institute of Science, Technology \& Advanced Stud- \\ ies, Chennai \\ ${ }^{2}$ Dy.Director, IPR Cell, Vels Institute of Science, Technology \& Advanced Studies, Chennai \\ ${ }^{3}$ Dean \& Head, Department of Computer Applications, Gurunanak College, Chennai \\ *Corresponding authorE-mail: ravi.se@velsuniv.ac.in
}

\begin{abstract}
The process of fuzzy image pattern recognizes object found in images by using the methods of fuzzy logic. Localization of object is al-so done. Fuzzy segmentation templates and operators, which fetch a large number of alternatives, constitute methods used in the method of fuzzy logic. Imperfect and imprecision of the input images and the templates images are in the consideration of fuzzy pattern matching and later incorporated in the matching process. This paper contemplates two methods one for fuzzy pattern and the other for the optimizing the matching scheme with a genetic algorithm. The process of optimization has its objective, in finding the location of reliable feature from a set of calibrated images through a simultaneous optimization of the templates and the segmentation function. Optimization has demonstrated and resulting a superior abstraction of the matches for an unobserved sample images and a good performance to the common method of pattern matching.
\end{abstract}

Keywords: Fuzzy logic; Genetic Algorithm; Image Pattern Matching; Image Segmentation; Object Recognition and Localization; Templates.

\section{Introduction}

The uses of pattern matching are found in the areas of recognition and localization of the objects in images. The other methods for object recognition is known to be more efficient computationally do exist. (e.g. invariant moments). But pattern matching is considered a competitive method for object localization. This process computes analogies between the input images and templates images or models, which are open to transform like rotation and translation.

Imperfects in logical reasoning are the parts of fuzzy logics, which have found application in image processing in many ways. The objective of segmentation is the division of pixels into homogeneous region that exhibiting similarity, i.e. crisp sets. In proper sequence of the Fuzzy segmentation bisects the pixels onto fuzzy sets, where each pixel to be a part in the regions of images for many sets.

Application of fuzzy logic has been seen in image enhancement and edge detection meant for the improvement of robustness against noise. Fuzzy logic in addition, affords a mean for linguistic expression of image processing. Operations have succeeded in developing a face detection algorithm which makes use of fuzzy pattern matching based on fuzzy analogy relation on two term basis. Fuzzy morphology is a model of explaining the binary image processing operators have been in fuzzy field.

Genetic Algorithm (GA) finds an application in every step and has seen in pattern recognition and image processing [12]. The fuzzy pattern matching system attains recognition by optimizing the membership functions using GA to reach the highest set of fuzzy entropy among the three sets of segmentation. GA also used for stereo vision matching. The GA's advantages have been availed in multi criteria optimization with simultaneous optimization of disparity smoothness and similarity matching.

An algorithm has been developed for detecting faces on the basis of edges and ellipse matching (yokoo et al). This involves smoothening of the binary edge to a multilevel image and matching of GA generated ellipses using the similarity of pixel-wise metric.

This paper presents an innovative technique of an optimization of fuzzy image pattern matching using genetic algorithm. Section [2] deals with the introduction of a fuzzy pattern matching scheme. In section 3 has its subject matter of a cost function for global optimization of the matcher. The testing method and comparison beside the general pattern matching modus formulated by the coefficient of Pearson correlation in section [4] and [5].

\section{Fuzzy pattern matching}

Each class of pattern matching exhibits the features of paradigm vector and the use of similarity measure lies in comparison of given paradigm patterns. 


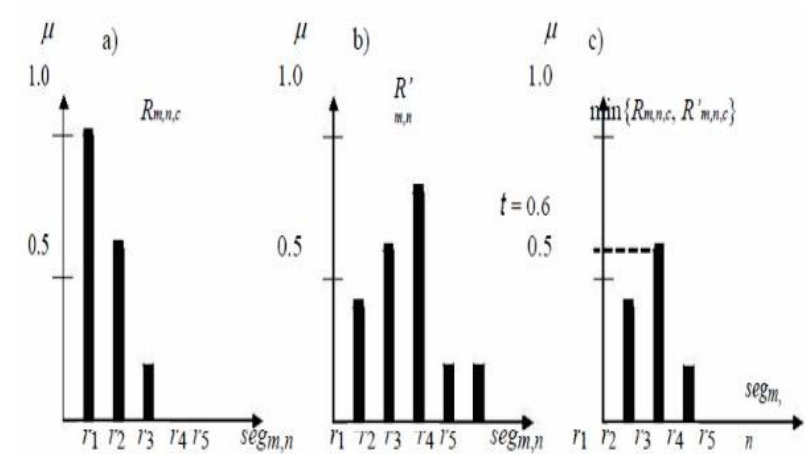

Fig. 1: A) A Feasible Deductive Segmentation, B) Fuzzy Segmentation Realized for Pixels (M, N)C) Accurate Analysis of Fact Formation That Segment Isrm,N.

This paradigm vector is a proverbial grey scale pattern image while correlation is the similarity metric in an image analysis.

$\mathrm{g}=/ \mathrm{cfw}$

$\mathrm{Sf} / \mathrm{sw}$

in which ' $\mathrm{c}$ ' is covariance of theimage ' $\mathrm{f}$ ', templates ' $\mathrm{w}$ ' and ' $\mathrm{s}$ ' is a standard deviation. (Gonzalez et al,).

The general paradigm vectors that are matched to image segmentation are binary pattern images. (e.g.) Minimum distance metrics are also used.

Pattern matching does not work well for multiclass object recognition when testing is done for all transformation combination. Object localization pattern matching is feasible, specifically in case of restriction to the search space for intervals of small magnitude in respect to a few parameters. There is a significant reduction in the computational cost.

The FPM technique is an extension of traditional method in pattern matching with some modifications .It is a set of varied resemblance which has fuzzy similarity relations and fuzzy reasoning .It is possible to operate FPM either on fuzzy segmented images or raw color / grey scale images.FPM is considered as more robust against noise and distortion compared to the classical pattern. Added to this, there is the resemblance of fuzzy methods to human reasoning, with the frequent consequence of linguistic rules that are known for intuitive understanding.

The FPM strategy depends on this paper is based on the segmentation of fuzzy similarity relations and fuzzy reasoning. The former has the goal of dividing the output image with many similar parts as fuzzy sets where every pixel being a member of any one particular region characterized by distinct identical membership class The Fuzzy segmentation comprises fuzzy clustering, fuzzy thresholding, and fuzzy reasoning at a later stage; it can be based on color, borders, texture etc.

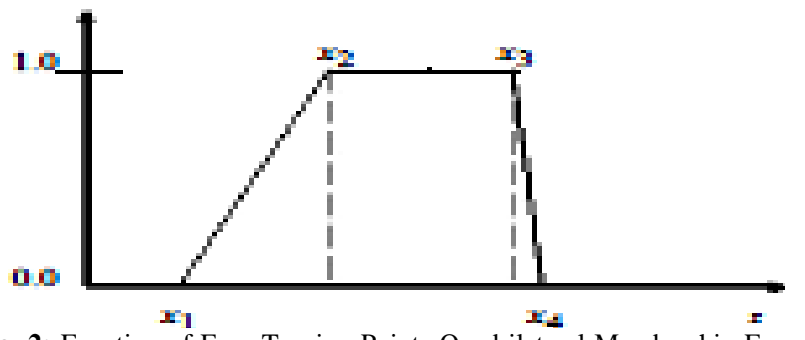

Fig. 2: Function of Four Turning Points Quadrilateral Membership Function Performed for Fuzzy Segmentation.

Fuzzy reasoning is the basis of FPM technique on fuzzy segmented images. It is based on the presumption of a target object class for a spatial pattern of segmented regions. The template reduced to a form of simplicity in the map of the desired segmented fuzzy sets.

A fuzzy rule core forms the template, wherein every pixel has an exclusive rule in implying the target class. The single line rule implies the firing strength, i.e., the entire region is the membership grade of the pixel in which the subject matter is the desired segmented fuzzy set. All the rules mean the aggregation of the overall truth.

The single line rule is:

IF segm,nis Rm,n,cTHEN class is Cm,n,c

Wherein $\operatorname{seg}_{m, n}$ represents the ROI for the segmentation of the pixel (m, n)in fuzzy set and $R_{m, n, c}$ is the membership function of feasible deductive segmentation.

Considering the fuzzy sets as R and seg, where the accurate analysis solves the firing strength which in turn complies the matching degree between the given and the desired MF's. The Fig. 1 explains how the accurate analysis is implied to a five class of fuzzy segmentation matching.

The template has the ability to include additional information in more complicated fuzzy pattern matching schemes. For example, every pixel may have a weight that corresponds to the pixels reliability in additions to the fuzzy connectives on a pixel.

\section{Genetic algorithm}

Effort is required for a through modeling of the problem where genetic algorithms find application to any specific field. Modeling comprises the choice of model free parameters, their genetic algorithm and creating an objective for optimization function.

\subsection{Trial coding}

The FPM technique used in this paper comprises the color based segmentation and implication of fuzzy. This model is a quite simple model and has complexity in solving the problem. The optimization of FPM method to one target class at any time with only one subject requiring localization in the presence of general segmentation rule and one template to be optimized and coded.

The degree of optimization depends on the simplicity of the model for faster and more reliable.This explains the absence of free parameters found in the Genetic Algorithm model, which provides the reason for the arbitrary choice of some parameters for the satisfactory results.

\subsubsection{Segmentation}

The Segmentation occurs based on the significant value of HueSaturation-Brightness (HSB). The fuzzy threshold procedure known for its global features finds application in segmentation of the input image that may go through pre-processing into two complementary fuzzy regions which could fall into two categories, viz, Foreground (FG) and Background (BG).Despite the corresponding strategy has no such differentiation. Periodically the inherent grouping is likely to indicate the regions vice-versa. The segmentation applicable on to the foreground is

IF hm,nis Handsm,nis Sandbm,n

THEN segm,nis FGisB

Whereas $h_{m, n}, s_{m, n}$, and $b_{m, n}$ are the hue, saturation and brightness of the input image pixel $(\mathrm{m}, \mathrm{n})$ respectively.

Reichenbach's conjunction multiplication has been used for AND operator. In this case, the fuzzy segmentation closure variable $\operatorname{seg}_{\mathrm{m}, \mathrm{n}}$ used to express the two values fg or bg for each pixel. AFG fuzzy set follows the consequences in a typical set as $\mathrm{FG}=\{(\mathrm{fg}$, $1.0),(b g, 0.0)\}$, where the Fig 3 illustrates the interference of an entire fuzzy set.

Considering a value of $\mathrm{seg}_{\mathrm{m}, \mathrm{n}}$ validatesthe extraction of foreground and background from a fuzzy sets. For instance, the fuzzy segmentation set describes an area for the FG is achieved through the collection of membership grades for seg $=$ fg into another fuzzy set: $\mathrm{seg}_{\mathrm{r}}=\mathrm{fg}=\left\{\left((0,0), \mathrm{m}_{\mathrm{fg}}\left(\operatorname{seg}_{0,0}\right)\right), \ldots \ldots,\left((\mathrm{m}, \mathrm{n}), \mathrm{m}_{\mathrm{fg}}\left(\operatorname{seg}_{\mathrm{m}, \mathrm{n}}\right)\right), \ldots ..\right\}$, whereas $\mathrm{m}_{\mathrm{fg}}\left(\mathrm{seg}_{\mathrm{m}, \mathrm{n}}\right)$ denotes the membership grade.

The fuzzy segmentation set outlines a 2D grid, which can be detected through changing the values of intensity. 
Considering the complementary features of the segmented regions and the membership grades for background can be obtained by negation.

$\operatorname{mbg}(\operatorname{segm}, n)=1-\operatorname{mfg}(\operatorname{segm}, n)$

Modeling the membership function (MF) $\mathrm{H}, \mathrm{S}$ and $\mathrm{B}$ is required for coding the segmentation rule. The choice of trapezoid MF's initiative to four free parameters per membership function (Fig.2) on the premise that the high and low levels is expressed at 1 and 0 individually. The four turning pinpoints should comply the discrepancy on $\mathrm{x}_{1} \S \mathrm{x}_{2} \S \mathrm{x}_{3} \S \mathrm{x}_{4}$. Hence the points which are relative coding are preferable to keep away forbidden trials. The second turning point $\mathrm{x}_{2}$ was specified as a perfect spot. The remaining parts are complying with:

$\mathrm{X} 1=\mathrm{x}_{2}-\mathrm{D}_{1}, \mathrm{x}_{3}=\mathrm{x}_{2}+\mathrm{D}_{2}, \mathrm{x}_{4}=\mathrm{x}_{2}+\mathrm{D}_{2}+\mathrm{D}_{3}$

The four resultant parameters are genetically coded as $\mathrm{x} 2, \mathrm{D}_{1}, \mathrm{D}_{2}$, and $\mathrm{D}_{3}$. Four bits have been preserved for every parameter and following this, they have $2^{4}=16$ different values which extends tothe input parameters of $\mathrm{H}, \mathrm{S}$, and $\mathrm{B}$. Genotype representation for $\mathrm{x}_{2}$ may be considered as $\mathrm{x}_{2}=0110$. The standardized composition might be $6 / 15=0.4$. The Membership Function modeling schemes pitfall lies in $\mathrm{x}_{\mathrm{i}}$, where the parameters are having different kinships. Particularly, $\mathrm{x}_{4}$ depend upon the remaining free parameters $\left(\mathrm{x}_{2}, \mathrm{D}_{2}\right.$, and $\mathrm{D}_{3}$ ).

\subsubsection{Template}

Simplicity in the template coding to the extent possible in the means for avoiding free parameters. This is the reason why the template of each pixel has one free parameter which decides the selected two different segmentation regions or the total omission of the pixel.

As a consequence, each pixel goes through modeling by two bits. These compositions of two bit are earmarked for omission and the segmentation for fuzzy set.

The MF's of $R_{m, n, c}$ in eq. (2) is what actually modeled, $C_{m, n, c}$ was adhered to 1 for the target class (class $=1$ ) and 0 for the opposite class (class $=$ unknown)respectively. The substitute for $R_{m, n, 1}$ are adhered to Foreground $=\{[\mathrm{Fg}, 1.0],[\mathrm{Bg}, 0.0]\}$ as well as Background $=\{[\mathrm{Fg}, 0.0],[\mathrm{Bg}, 1.0]\}$. It is possible to model the rule of omission in it's entirely without knowing the effect on the conclusion. This case is therefore; deals with individually in a group of step. The fuzzy segmentation-matching rule comes into:

IF Segm,nis FgthenClass is C and .

IF Segm,nis BgthenClass is C

The Fig 3 illustrates the general decision making fuzzy system for a pixel $(\mathrm{m}, \mathrm{n})$. The expectation of the pixel belongs to the background where its membership grades are 0.4 on the fuzzy set and consequently abet offered by the pixel of the target class is rather low. Nevertheless, an aggregation of the general closure for a ROI is done for all the pixels.

For example, a small template is shown in Fig 4, a point to note that the Hamming distance from Foreground and Background to 'omit' is 1 . This helps effectively by optimizing the genetic operators, emphasized by mutation and crossbreeding.

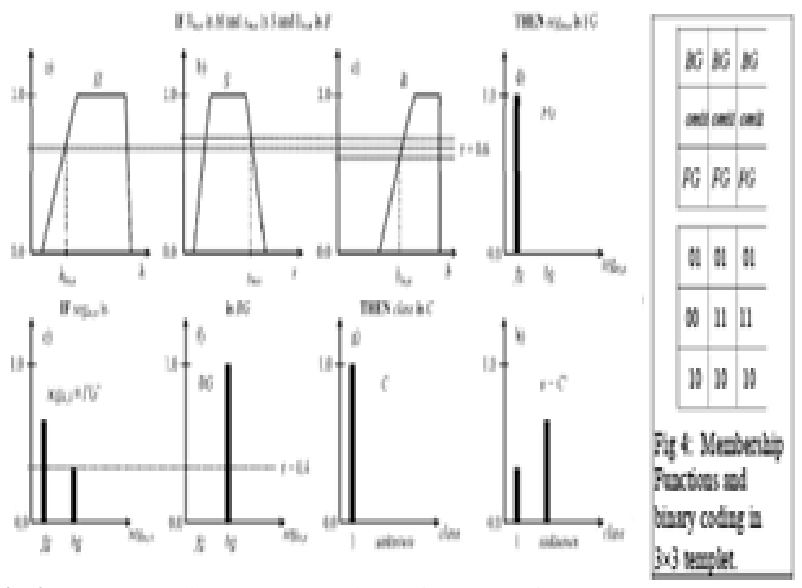

Fig.3: A Symbolic Representation of FPM Inference System. A to C) Ancestor Sectiond) Decisive Section of the Segmentation Rule. E) Postulate and Segmentation Result for the Classification Rule. F) Ancestor Section, G) Decisive Part of the Classification Rule and H) Classification Results

\subsection{Cost function}

The result of the scalar evaluation of a trial represents the objective function (cost and fitness). Instruction from the fitness function is the genetic algorithm driven to an optimum. In a task that involves multi-criteria optimization, aggregation of the distinct objectives is merged to a particular scalar.

However the cost function framework has a remarkable result in optimizing the speed and robustness. This means the cost function possess to do efficient guidance for GA to an optimal one.

Accuracy and robustness constitute the objectives for optimization of FPM, which implies standard localization and discrimination of improper position respectively. Euclidian distance is the yardstick for the measurement of accuracy done to the reference point $\left(\mathrm{m}_{0}\right.$, $\mathrm{n}_{0}$ ) from the best match considering an exact point for the desired object:

$\operatorname{dist}(\mathrm{y})=\sqrt{\left(\mathrm{m}-\mathrm{m}_{0}\right)^{2}+\left(\mathrm{n}-\mathrm{n}_{0}\right)^{2},} \operatorname{Max} \mathrm{m}, \mathrm{n}\left\{\mathrm{m}\left(\mathrm{ym}_{\mathrm{m}, \mathrm{n}}\right)\right\}$

Whereas $y=2 D$ finale fuzzy set.

The perception of optimization means increasing $(\max )$ the matching degree at the referral point and decreasing (min) it to another point.

But there is a strong, spatial correlation in image seen in the degree of match. Consequently, the needs to take the minimization criteria outside are appropriate distance from the reference point. The maximum best match (overall match) and the maximum outside match for a specific interval $r$ defines a Discrimination Factor (DF)

$\mathrm{DF}(\mathrm{y})=\max _{\mathrm{m}, \mathrm{n}}\left\{\mathrm{m}\left(\mathrm{y}_{\mathrm{m}, \mathrm{n}}\right)\right\}-\max _{\mathrm{m}, \mathrm{n}}\left\{\mathrm{m}\left(\mathrm{y}_{\mathrm{m}, \mathrm{n}}\right)\right\} \cdot(\mathrm{m}, \mathrm{n})-(\mathrm{m} 0, \mathrm{n} 0)^{3} \mathrm{r}$

$\operatorname{DF}(y ; a)=a \max _{m, n}\{m(y m, n)\}-(2-a) \max _{m, n}\left\{m\left(y_{m}, n\right)\right\}(m, n)-$ $\left(\mathrm{m}_{0}, \mathrm{n}_{0}\right) \mathbf{I}^{\mathrm{3}} \mathrm{r}$

By the selection of $a=[1]$, the eq. (9) diminishes to eq. (8). A common method of emphasizing the fuzzy connectives and fuzzy logic uses a combined criterion of Discrimination Factor and the maximum matching degree. Linguistic expression is done as the objective being to maximize both the Discrimination Factor and the maximum match (or vice versa) refers to the Certainty Factor $(\mathrm{CF})$ and it is given by:

$\mathrm{CF}(\mathrm{y})=\max _{\mathrm{m}, \mathrm{n}}\{\mathrm{m}(\mathrm{ym}, \mathrm{n})\}^{*} \mathrm{DF}(\mathrm{y})$

Whereas* is a fuzzy connective.

The best fitness function is an integration of the modified DF and a distance term: 
$€(y)=c_{0}-c_{2} \cdot D F(y ; a), \operatorname{dist}(y)<d_{1} c_{0}+c_{1}+c_{1}$. dist $(y)-$ $c_{2} \cdot \operatorname{DF}(y ; a), d_{1}<\operatorname{dist}(y)<d c_{0} \cdot \operatorname{dist}(y)-c_{2} . D F(y ; a)$, otherwise

Whereas $c_{i}$ and $d_{i}$ are inconsistent manner.

The minimization of the cost function is necessary; the reason for this decrease is in the cost function as the distance and the DF increases. There is a gradual decrease in the effect of distance requires the near referral point. The Distance belowd $d_{1}$ in the fitness function is omitted by considering the best enough and the referral points possess an error in the determination of a few pixels. Guidance to an optimum distance is the first thing followed by emphasizing on the Discrimination Factor.

\section{Analysis for nose and iris}

The noise and Iris location of the human have been chosen for the trial case, considering the spontaneous searching on the nature of the problem and the peer orientation determination has many manifold applications. For example consider the human machine interrelation.

The measurement of Horizons between the irises to the center line of the nose and the horizon between the irises provides a solution to the problem of peer orientation in related to the skull, but it requires calibration. The solutions to the orientation of the skull could be explained with the spatial diversity interrelationship. The gestureof the eyes are small, there is difficulty in finding the center line of the nose, when the images are acquired though a webcam makes the process difficult because oflow resolution in webcam.

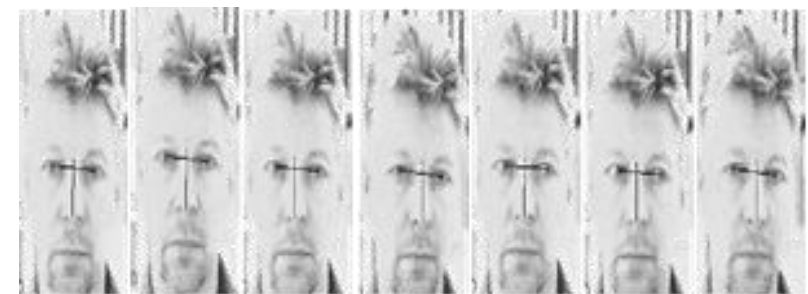

Fig. 5: Estimation of the Referral Spots for Calibration and Test Images (in Random Sequence).

The trial images have been taken almostfrom an identical point, horizon, rotation, angle and lighting conditions enable concentration on variation of only the translation when matching is done and therefore reducing the complete of computations. For finding a simple and faster algorithm, manual step of face localization, despite various algorithms for the purpose have been outlined in this work. Hence the nose localization of ROI has a finite approach to the face area subsisting of $120 \times 180$ pixels.

A search in a completely automated application would be hierarchical. For example though subsequent location of nose, right eye, left eye and face through use of a prior understanding for every step. The current objective is to study the optimization of FPM inclusion of three referral images with varying directions of sight has been done in the calibration set with four trial set. The determination from the referral points of the nose are illustrated in the fig 5.

The dimensions of the template seen in all trial runs are: $42 \times 52$ for the nose and $10 \times 6$ pixels for iris .The templates for recognition of nose consist of $3 \times 3$ structural size in equal MF's is to minimize the free parameters. Accordingly the nose template holds $14 \times 18$ $=252$ free parameters and the iris template holds 60 . The fuzzy segmentation requires 4 membership functions where every 4 parameters require 4 bits and 2 bits for every template parameter. The total number of bits requires for optimization is $4 \times 4 \times 4+252 \times 2=268$ bits for localization of nose and $64+60 \times 2=184$ bits for localization of iris.

Definition of one FPM result is done for the cost function in eq (11). The cost functions for three calibration images were the maximum of cost function evaluation $\mathrm{e}_{\mathrm{I}}$ for three items, in which the corresponding results to the case as worst.
However evaluating the cost function was done only when the results of temporary fitness was seen beneath 1000 and better in comparison with the worse fitness in a selected group. This is significant in reducing the complexity involved in computation at the initial stages.

Genetic operators had various manifestations, viz, bitwise mutation, uniform crossover and one point cross over.The small population size of (50) and 20 offspring were created for every generation from the elite through use of roulette wheel selection. The population size was small corresponding to the local strategy that could be fast, but inconsistent. There is always a balancing done between speed and reliability. Following a few trial runs, the parameters of a cost function have been fixed as; $\mathrm{d}_{1}=2, \mathrm{~d}_{2}=2, \mathrm{c}_{0}=$ $1000, c_{1}=50, c_{2}=1000$ and $a=1$.Radius $r$ is the semi rim of the template.

Considering the pattern matching correlation as a traditional process (eq. 1) was used as a referral device. A template size which is identical to the fuzzy pattern matching template was designed by cropping the RGB channels from one image around the target location, while the remaining images were used for trial purpose.

\section{Results}

An experimental result carries the reflecting measures of accuracy and robustness related to the optimization of fuzzy pattern matching system and statistics of optimizing genetic algorithm.Discussion more particularly of the FPM results on generalization ability.

\subsection{Localization of nose}

The localization of nose optimization of FPM system was carried out by the two GA runs of 500 generation. The run 1 are used for the raw images, while in run 2 the preprocessed images are preserved with uniform color.

Table 1 provides a result summary of three calibration and four trial images where Dist is the pixels location errors. The results of the optimal matcher indicates good generalization as the unseen test images whose pose scale and lighting conditions are almost exact, with variation in the pose of the eyes.

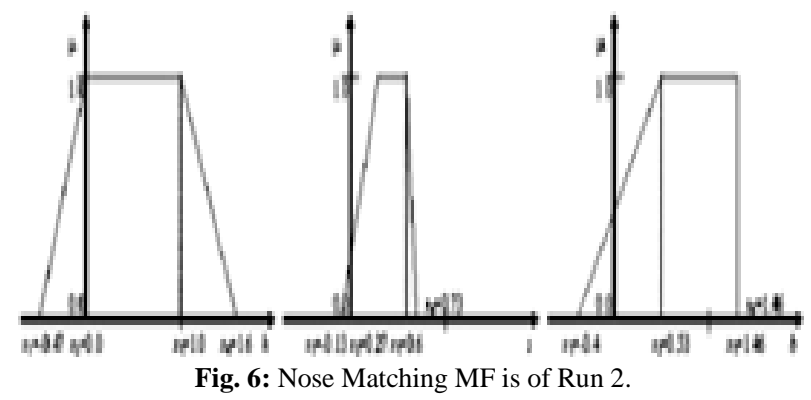

The ability for generalization, in all the probability was established from the existing segmentation (feature creation) and an optimizing template (feature selection).The pixels selection is done by GA with clear information on the calibration images.Pixels present in the test images have features that are interimage-variant.

The reference method (correlation) is also an organized generalization for the trial images. The location error was quite small while the DF was impecunious. The potential of causing a sever dislocation under a noisy environment.

An illustration of the optimized membership functions of run2 is provided by fig 6 . The absence of any role for hue in segmentation which is evident is due to its feature being a constant 1 in $[0,1]$, in the normalized hue value range. The marginal brightness impact is seen on the nose. Its nearby brightness values below 0.53 are rare. In particular, pixels around the nose are $0.18 \S \mathrm{s} \S 0.27$ and pixels everywhere around the noseusuallyhave the features ofs $>0.27$. 
Table 1: Results for Nose Matching After 500 Generations. Dist Is the Pixels Location Error, Max the Maximum Degree of Match, and DF is stated by Eq. (8). 'Remarks' is the Reference Method in Eq. (1)

\begin{tabular}{llllllllll}
\hline & \multicolumn{3}{c}{ run 1 } & \multicolumn{4}{c}{ run 2 } & \multicolumn{3}{c}{ Remarks } \\
\hline Image & Dist & Max & DF & Dist & Max & DF & Dist & Max & DF \\
Cali 1 & 2 & 0.93 & 0.29 & 1 & 0.85 & 0.25 & 0 & 0.99 & 0.18 \\
Cali 2 & 2 & 0.93 & 0.29 & 1 & 0.85 & 0.25 & 1 & 0.95 & 0.16 \\
Cali 3 & 1 & 0.94 & 0.31 & 0 & 0.85 & 0.25 & 1 & 0.96 & 0.17 \\
Trial1 & 2 & 0.91 & 0.27 & 2 & 0.86 & 0.24 & 1 & 0.91 & 0.17 \\
Trial2 & 3 & 0.92 & 0.28 & 2 & 0.84 & 0.24 & 1 & 0.96 & 0.16 \\
Trial3 & 4 & 0.89 & 0.20 & 0 & 0.85 & 0.19 & 1 & 0.95 & 0.16 \\
Trial4 & 4 & 0.86 & 0.20 & 2 & 0.83 & 0.18 & 1 & 0.94 & 0.16 \\
\hline
\end{tabular}

The Fig.7illustrates the optimizing template, segmentation result, matched image and the matching degree. On optimizing the spatial pattern of the template enables location of the contributing pixels and the borders of the nose with high contrast, where the regions of the upper jaw, nostrils and the edges of eyes. In sharp contrast, the slopes of a nose side are unreliable to some extent as a result of sensitivity to lighting conditions and poor contrast.

Fig.7d) illustrates the FPM techniques discrimination and spatial autocorrelation. An extent of match has a circular correlation pattern everywhere. Nevertheless, the matching degree outside the neighborhood is more inferior specifying good discrimination.

\subsection{Localization of iris}

The irises location of the eyes was done using optimization of FPM in addition to the nose. The right eye results (from the reader's view) are provided.

The nine similar images in the nose localization were used, but the calibration images were selected to ensure inclusion of eye's different poses. The table-2proves the good generalization, for the two GA runs of 150 generations but the DF's in the nose localization are lower, which might be due to the small size template. So, the probability of having better matches at random is higher.

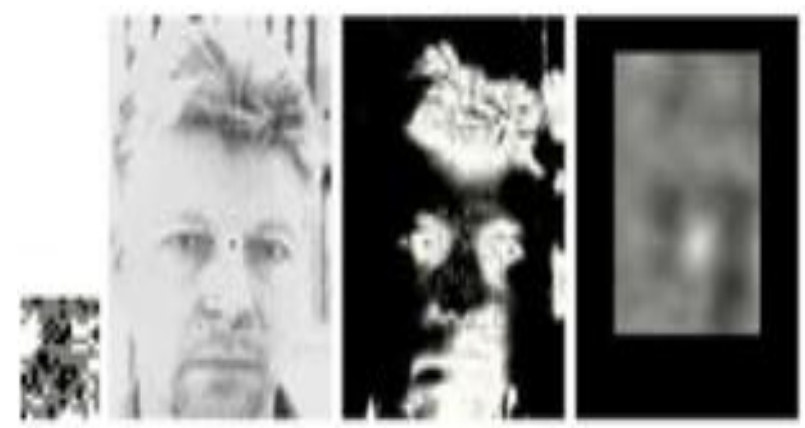

Fig. 7: Results of Run 2 (Uniform Scaling). A) Optimized Template, B) Overlaid Template for the Best Match Position) Segmented Image Using FPM and D) Degree of Match (I.E. Black Area = Uncomputed).

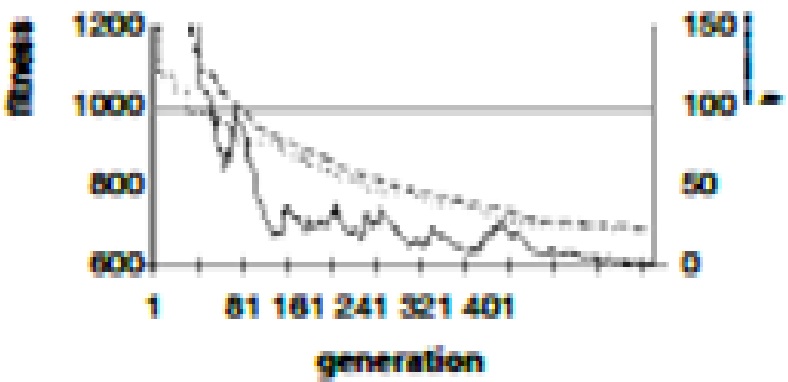

Fig. 8: The Best Transforms (Dotted Line), Worst Fitness (Dashed Line) in the Selected Group of Nose Run 1.Fitness Array = Solid Line.

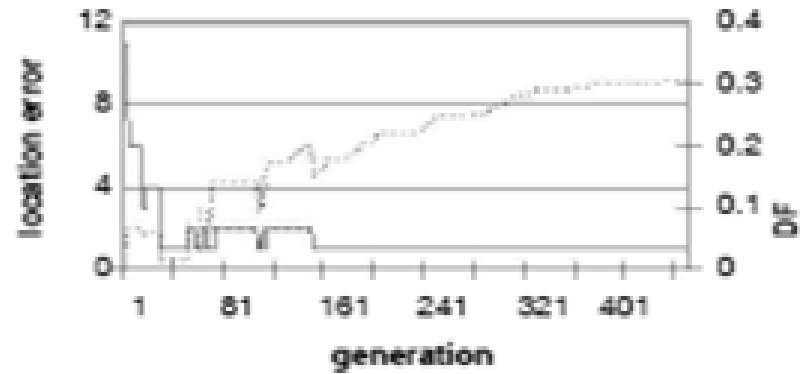

Fig. 9: Transform of Location Error (Solid Line) and DF (Dotted Line)

There are [5] of 6 failures in the trial images. The negative DF was analyzed by deducting the maximum degree of match from the desired location degree of match, which is clearly expressed with an example.

Analysis of MF's in Fig 10 and the HSB values of the image suggested the brightness values of the images as a dominant feature. Actually, the turning point $b=0.6$ appears to be a good selection to separate iris and pupil $(b<0.6)$ from the context $(b>0.6)$. The white of the eye recognize to a crucial point, on varying feature as the eye turns.

The segmentation results are identical despite differences in the segmentation membership functions. The conclusion of the brightness is being the dominant features as membership function as the only similar ones is therefore validated. Segmentation results also reveal the separation of the white of the eye from an iris. This lends credibility to the presumption of the selection of calibration images with different eye poses compelled the selection of such MF's.

\section{Conclusion}

The manner in which fuzzy logic is used for fuzzy pattern matching segmentation has been demonstrated.Uncertainties inherent in measurement data like images have been incorporated by fuzzy pattern matching in computational steps and results. The 2D output of fuzzy set provides a clear indication of the possibilities of the different locations of the target object.

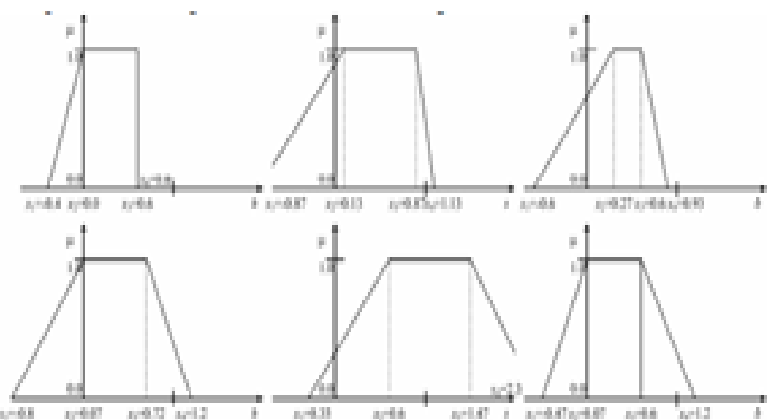

Fig. 10: $M F$ is for Iris Matching of Run 2 Upper and Run 2lower.

Table 2: Iris Matching Results after 150 Generations

\begin{tabular}{|c|c|c|c|c|c|c|c|c|c|}
\hline & & \multicolumn{2}{|c|}{ run 1} & \multicolumn{3}{|c|}{ run 2} & \multicolumn{3}{|c|}{ Remarks } \\
\hline Image Dist & & Max & DF & Dist & $\operatorname{Max}$ & DF & Dist & Max & DF \\
\hline Cali 1 & 2 & 0.95 & 0.12 & 2 & 0.92 & 0.15 & 0 & 1.00 & 0.05 \\
\hline Cali 2 & 2 & 0.91 & 0.12 & 1 & 0.95 & 0.18 & 1 & 0.96 & 0.02 \\
\hline Cali 3 & 2 & 0.96 & 0.13 & 2 & 0.94 & 0.16 & 72 & 0.79 & -0.14 \\
\hline Trial1 & 2 & 0.90 & 0.08 & 3 & 0.95 & 0.16 & 85 & 0.87 & -0.08 \\
\hline Trial2 & 4 & 0.96 & 0.15 & 2 & 0.97 & 0.17 & 46 & 0.89 & -0.06 \\
\hline Trial3 & 3 & 0.96 & 0.11 & 2 & 0.94 & 0.16 & 70 & 0.92 & -0.02 \\
\hline Trial4 & 3 & 0.96 & 0.09 & 2 & 0.90 & 0.06 & 65 & 0.89 & -0.05 \\
\hline
\end{tabular}

The study illustrates, the three membership functions in HSB color space to perform a fuzzy segmentation using the fuzzy pattern matching method. The template comprises the desired spatial patterns of the segmented pixels. Optimization of both the membership functions and the template was done by using a stochastic search study and a genetic algorithm. The results indicate good 
generalization of the optimized FPM system to the trial images. In fact the offset against the set of images were small, not seeing much noise. However, the innovative method has performed better than the traditional correlation pattern matching extremely in many instances.

\section{References}

[1] W-B. Tao, J-W. Tian, J. Liu.'Image segmentation by three-level thresholding based on maximum fuzzy entropy and genetic algorithm.'Pattern Recognition Letters, 24: 3069-3078, 2003.

[2] R.C.Gonzalez, R.E.Woods. Digital Image Processing. Second edition. Prentice-Hall, New Jersey, 2002.

[3] M Sonka, V. Hlavac, R. Boyle. Image Processing, Analysis, and MachineVision. Second edition. Brooks/Cole Publishing Company, USA, 1999.

[4] K.M.Passino, S. Yurkovich. Fuzzy Control, Addison-Wesley, California, 1998

[5] D.B. Russakoff, T. Rohlfing, C.R. Maurer Jr. Fuzzy segmentation of X-ray fluoroscopy image. Medical Imaging 2002: Image Processing. Proceedings of SPIE 2684, 2002.

[6] W-B.Tao, J-W.Tian, J. Liu. Image segmentation by three-level thresholding based on maximum fuzzy entropy and genetic algorithm. Pattern Recognition Letters, 24: 3069-3078, 2003.

[7] B. Alhadidi, H. N. Fakhouri, and O. S. Al Mousa, cDNA Microarray Genome Image Processing Using Fixed Spot Position, American Journal of Applied Sciences, 3, 1730-1734, 2006.

[8] .N.Giannakeas and D.I. Fotiadis, Image Processing and Machine Learning Techniques for the Segmentation of cDNA Microarray Images, Handbook of research on advanced techniques in diagnostic images and biomedical application, 2008.

[9] D.Ravikumar, Dr.Arun Raaza, Dr.V.Devi "Computational Analysis of Microarray Image" Journal of Advanced Research in Dynamical and Control Systems, 09- Issue No: 5, 67-75, July-17

[10] National Human Genome Research Institute, http://www.genome.gov, 2009.

[11] D.B. Russakoff, T. Rohlfing, C.R. Maurer Jr.Fuzzy segmentation of X-ray fluoroscopy image. Medical Imaging 2002: Image Processing.Proceedings of SPIE 2684, 2002.

[12] Dharmarajan, K., and M. A. Dorairangaswamy. "Discovering Student E-Learning Preferred Navigation Paths Using Selection Page and Time Preference Algorithm." International Journal of Emerging Technologies in learning (iJET) 12.10 (2017): 202-211. 